\title{
OXY-HEMOGLOBIN DISSOCIATION CURVES OF WHOLE BLOOD IN ANEMIA
}

By DICKINSON W. RICHARDS, JR., AND MARJORIE L. STRAUSS

(From the Department of Medicine, College of Physicians and Surgeons, Columbia University, and the Presbyterian Hospital, New York City)

(Received for publication October 3, 1926)

Although the oxy-hemoglobin dissociation curves of normal whole blood have been extensively studied for many years $(1,2,3)$, there has been relatively little investigation of these curves in the blood of abnormal subjects.

Meakins, Dautrebande and Fetter (4), in their work on circulatory stasis in 1923, published some oxygen dissociation curves of patients with cardiac decompensation, showing that at or near $40 \mathrm{~mm}$. $\mathrm{CO}_{2}$ tension, these curves did not deviate appreciably from those of normal blood. Stadie and Martin (5), investigating carbon monoxide hemoglobin and oxy-hemoglobin relations, included one oxygen dissociation curve of a patient with pernicious anemia. This was at 40 $\mathrm{mm} . \mathrm{CO}_{2}$ tension, and was an apparently normal curve. Odaira (6) stated that in severe anemia the oxygen curves were lowered, but did not state at what $\mathrm{CO}_{2}$ tension or serum $\mathrm{pH}$ these curves were determined.

The present investigation represents a study of the oxy-hemoglobin dissociation curves of the whole blood of several subjects with anemia from various causes, and of one with advanced polycythemia vera. It comprises the following: (a) at serum $\mathrm{pH}$ (or $\left.\mathrm{pH}_{\mathrm{s}}\right) 7.44\left(\mathrm{CO}_{\mathbf{2}}\right.$ tension approximately $40 \mathrm{~mm}$.), points on the $\mathrm{O}_{2}$ dissociation curves of five primary anemias, two secondary anemias, and one polycythemia vera; (b) at $\mathrm{pH}_{8} 7.64\left(\mathrm{CO}_{2}\right.$ tension approximately $20 \mathrm{~mm}$.), points on the curves of three primary and two secondary anemias; (c) at $\mathrm{pH}_{\mathrm{s}} 7.24\left(\mathrm{CO}_{2}\right.$ tension approximately $80 \mathrm{~mm}$.), points on the curves of one primary and one secondary anemia. A control curve of the blood of one of us was also done at each of these $\mathrm{CO}_{2}$ tensions. 


\section{METHODS}

Blood was drawn from an arm vein, with stasis of one minute or less, into a container with enough neutralized dried potassium oxalate, and dried sodium fluoride to make a concentration of approximately 0.2 per cent of the former and 0.1 per cent of the latter. Tonometers of $300 \mathrm{cc}$. capacity were filled with the desired $\mathrm{CO}_{2}$ and $\mathrm{O}_{2}$ mixtures by the manometer method outlined by Van Slyke, $\mathrm{Wu}$ and McLean (7). Five cubic centimeters of blood were introduced into each tonometer. Two tonometers were then put into a water bath at $38, \pm 0.2^{\circ} \mathrm{C}$., and rotated for forty minutes or more. The other tonometers were put in the ice box, and equilibrated later. The gases in the equilibrating tonometers were brought to atmospheric pressure at $38^{\circ}$, by allowing excess gas to escape at the beginning of the equilibration and again after about ten minutes of rotating. The effect of equilibrating the blood in the tonometers for longer than forty minutes was tested on the blood of one of us (see table 1, experiment of March 29th) at an oxygen tension of $20 \mathrm{~mm}$. There was no measurable change in the oxygen capacity of the blood in the tonometers after either two hours' or four hours' rotation. After equilibration the blood was withdrawn directly into 1 cc. stopcock-pipettes, and then transferred for oxygen or $\mathrm{CO}_{2}$ determination to a Van Slyke-Neill constant volume apparatus. Samples of the tonometer gases were collected in gas sampling tubes and their $\mathrm{CO}_{2}$ and $\mathrm{O}_{2}$ contents determined later by the Haldane gas analysis apparatus. The above procedure is in general that of the "first saturation method" of Austin, Cullen et al. (8).

In two of the earlier experiments, the blood was collected from the tonometers into test tubes under oil. Under these conditions, however, the blood was found to absorb oxygen and lose $\mathrm{CO}_{2}$, especially if stirring was necessary, as was usually the case on account of the rapid settling of anemic blood. The transfer of blood directly to pipettes saved one step in manipulation; was easily accomplished by connecting three or four pipettes successively to the tonometer by a bent glass tube connection, and drawing the blood into them; and this method gave duplicate determinations that checked satisfactorily. A pipette full of blood could be left standing several minutes without measurably changing the $\mathrm{O}_{2}$ content.

Oxygen capacity determinations were in most cases made after equilibrating the blood in air at room temperature; occasionally in tonometers at $38^{\circ} \mathrm{C}$.; the proper value for dissolved $\mathrm{O}_{2}$ being applied in each case.

There was considerably greater difficulty in obtaining an accurate curve from a markedly anemic blood than from normal blood. This was partly because of the rapidity of settling of the red cells, and partly because of the magnification of errors in per cent oxygen saturation when the oxygen capacity was small. In the first two of our primary anemia curves at $40 \mathrm{~mm} . \mathrm{CO}_{2}$ tension, in which the blood after equilibration was collected under oil, our duplicate determinations did not check closely. In the succeeding experiments, a total of 61 points on abnormal blood curves were determined: duplicate determinations on two of them checked only to 0.4 volume per cent; four others to 0.3 volumes per cent; and 
four points were based on a single oxygen content determination. Duplicate measurements for the other points checked within the error of the method, 0.2 volumes per cent. Of 31 points on our control blood, three checked only to 0.4 volumes per cent, the rest within the error of the method. An exception is made of the case of W. B., (table 3), a secondary anemia whose major condition was myelogenous leukemia. His white blood count was 700,000; and his blood was found to diminish rapidly in $\mathrm{O}_{2}$ content on standing; so that we were compelled to use for our curve only the first oxygen measurement after equilibration of the blood, the first pipetteful of blood being transferred as rapidly as possible from the tonometer to a Van Slyke apparatus containing air-free ferricyanide solution:

\section{CORRECTIONS}

The form of the dissociation curve of oxy-hemoglobin has been shown, by Adair (9) and others, to depend primarily on the $\mathrm{pH}$ of the solution, although the content of bicarbonate and other electrolytes also influences the levels of the curves to some extent $(10,11,1)$. For the comparison of oxygen dissociation curves of the whole blood of different individuals, it would therefore probably be best to have all curves corrected to the same cell $\mathrm{pH}\left(\right.$ or $\mathrm{pH}_{\mathrm{c}}$ ). Such corrections can be made, as fairly good approximations, by the use of the Donnan ratio $r$, as developed by Van Slyke, Wu and McLean (7), if the $\mathrm{pH}^{\text {. }}$ and percentage oxygen saturation are known. When these corrections are worked out, however, using the data of Bock, Field and Adair (3), it is found that the differences between the curves at constant $\mathrm{pH}_{\mathrm{c}}$, at constant $\mathrm{pH}_{\mathrm{s}}$, and at constant $\mathrm{CO}_{2}$ tension, are small; and although the larger corrections are outside the limits of the experimental error, they are smaller than the recognized and as yet unexplained differences between the blood of different normal individuals. We have, therefore, in these curves simply determined the $\mathrm{pH}_{\mathrm{s}}$ of the oxygenated blood at the desired $\mathrm{CO}_{2}$ tension, 20,40 , or 80 $\mathrm{mm}$., and if this $\mathrm{pH}_{\mathrm{s}}$ value has differed by more than 0.04 from that of the standard normal curves, a correction has been applied to all the points on that curve. Four of the curves, one primary anemia, two secondary anemias, and one control blood, required such a correction. The corrections have been made in the $\mathrm{O}_{2}$ tension by interpolation, using the curves of Bock, Field, and Adair as standards.

The $\mathrm{pH}_{\mathbf{s}}$ was determined gasometrically, by the Henderson-Hasselbalch formula. The $\mathrm{CO}_{2}$ content of the oxygenated blood at the 
desired $\mathrm{CO}_{2}$ tension was determined by measuring the $\mathrm{CO}_{2}$ content (either whole blood or "true" serum) of the blood in the tonometer having the highest $\mathrm{O}_{2}$ tension. This blood was, in the various cases, from 90 to 98 per cent saturated with oxygen. The $\mathrm{CO}_{2}$ tension, as measured, varied usually a few millimeters from the exact value desired, i.e., 20, 40, or 80. A small extrapolation on the $\mathrm{CO}_{2}$ curve, with correction for oxygen unsaturation, then gave the $\mathrm{CO}_{2}$ content of fully oxygenated blood at the exact $\mathrm{CO}_{2}$ tension, with sufficient accuracy. In some of the determinations we measured the whole blood $\mathrm{CO}_{2}$ content and in others that of the "true" serum. In the former case the $\mathrm{pH}_{\mathrm{s}}$ was determined by the method outlined by Van Slyke, $\mathrm{Wu}$ and McLean (7), using their $\Delta \mathrm{pK}^{\prime}$ values. We used 6.13 as the $\mathrm{pK}^{\prime}$ value, and $\alpha_{\mathrm{CO}_{2}}=0.555$ per $\mathrm{Kg}$ of blood water for the solubility factor, as employed by Van Slyke, Hastings, Murray and Sendroy (12). These constants gave slightly different $\mathrm{pH}_{\mathrm{s}}$ values to our curves, and also to the curves of Bock, Field, and Adair, than when computed by using the constants of Van Slyke's earlier paper (7).

In as much as in our gas equilibrations we used the "first saturation method" of Austin, Cullen, et al. (8), the $\mathrm{CO}_{2}$ tensions in the tonometers were only approximately correct and the Haldane analyses after equilibration frequently showed the final $\mathrm{CO}_{2}$ tensions to be several millimeters from the desired tensions of 20,40 , or $80 \mathrm{~mm}$. A correction formula was developed on the basis of the empirical linear relation between $\mathrm{CO}_{2}$ tension and $1 / \mathrm{K}$ of Hill's equation, a relation which L. J. Henderson (13) found to be approximately true when applied to Barcroft's blood curves, and which he expressed in the formula: $\frac{\mathrm{p}_{2}+7.7}{0.014}=\frac{\mathrm{Hb}}{\mathrm{HbO}_{2}}\left(\mathrm{pO}_{2}\right)^{2.5}$. The correction formula which we have used is the same in principle as that used by Bock, Field and Adair, and is, for the $40 \mathrm{~mm}$. curves, as follows:

$$
\log \mathrm{p}_{\mathrm{o}_{20}}=\frac{1}{2.5} \log \left[(40+7.7)+2.5 \log \mathrm{p}_{\mathrm{o}_{2}}-\log \left(\mathrm{p}_{\mathrm{co}}+7.7\right)\right]
$$

where $\mathrm{P}_{\mathrm{O}_{20}}=\mathrm{O}_{2}$ tension at $40 \mathrm{~mm} . \mathrm{CO}_{2}$ tension

$\mathrm{p}_{\mathrm{O}_{2}}=\mathrm{O}_{2}$ tension as measured

$\mathrm{P}_{\mathrm{CO}_{2}}=\mathrm{CO}_{2}$ tension as measured. 
As a matter of fact, except in the cases of the larger corrections, especially those near 100 per cent oxygen saturation, little difference was found between the corrections, based on the above formula, and those found merely by interpolation, using as standards the curves of Bock, Field and Adair. The method of interpolation, being simpler, was therefore usually employed for the smaller corrections.

\section{DISCUSSION}

The data for all the curves are tabulated in tables 1, 2 and 3, and the points charted in figures 1 to 3 . The drawn curves in the figures, included for purposes of comparison, are reproduced from the data of Bock, Field and Adair, the continuous lines being from the blood of A. V. B. and the interrupted line from that of G. S. A. Clinical data in regard to the patients studied are given in table 4.

It will be seen that all the $\mathrm{pH}_{\mathrm{s}}=7.44$ curves fall fairly close to the normal ones, both for primary and secondary anemias. There is perhaps a tendency for the anemic curves to lie at a little lower level, especially in the upper part of their course, than the normals. The polycythemia curve shows no evidence of abnormality.

The points on the $\mathrm{pH}_{\mathrm{s}}=7.24$ curves also are fairly close to each other; the normal curve here, however, does not agree as well with that of A. V. B. We have no special comment to offer on this latter point, except that for some reason a smooth and accurate curve is, in general, more difficult to obtain at this high $\mathrm{CO}_{2}$ tension. Bock, Field, and Adair encountered the same thing in their work. In our final normal control experiment, all oxygen determinations were done in triplicate, and the average of the closest two values were taken for each point.

At the more alkaline reaction of $\mathrm{pH}_{8}=7.64$ there are obviously distinct differences between the curves of the individuals studied. The number of curves is, of course, small, and it is therefore quite possible that the variations in the levels of the curves simply represent variations that might occur between the oxygen dissociation curves of any small group of normal individuals. That such differences do occur has been known since Barcroft's early work (1). It is clear from an examination of figure 2, however, that our normal control curve is close to that of A. V. B., and that the anemia curves 


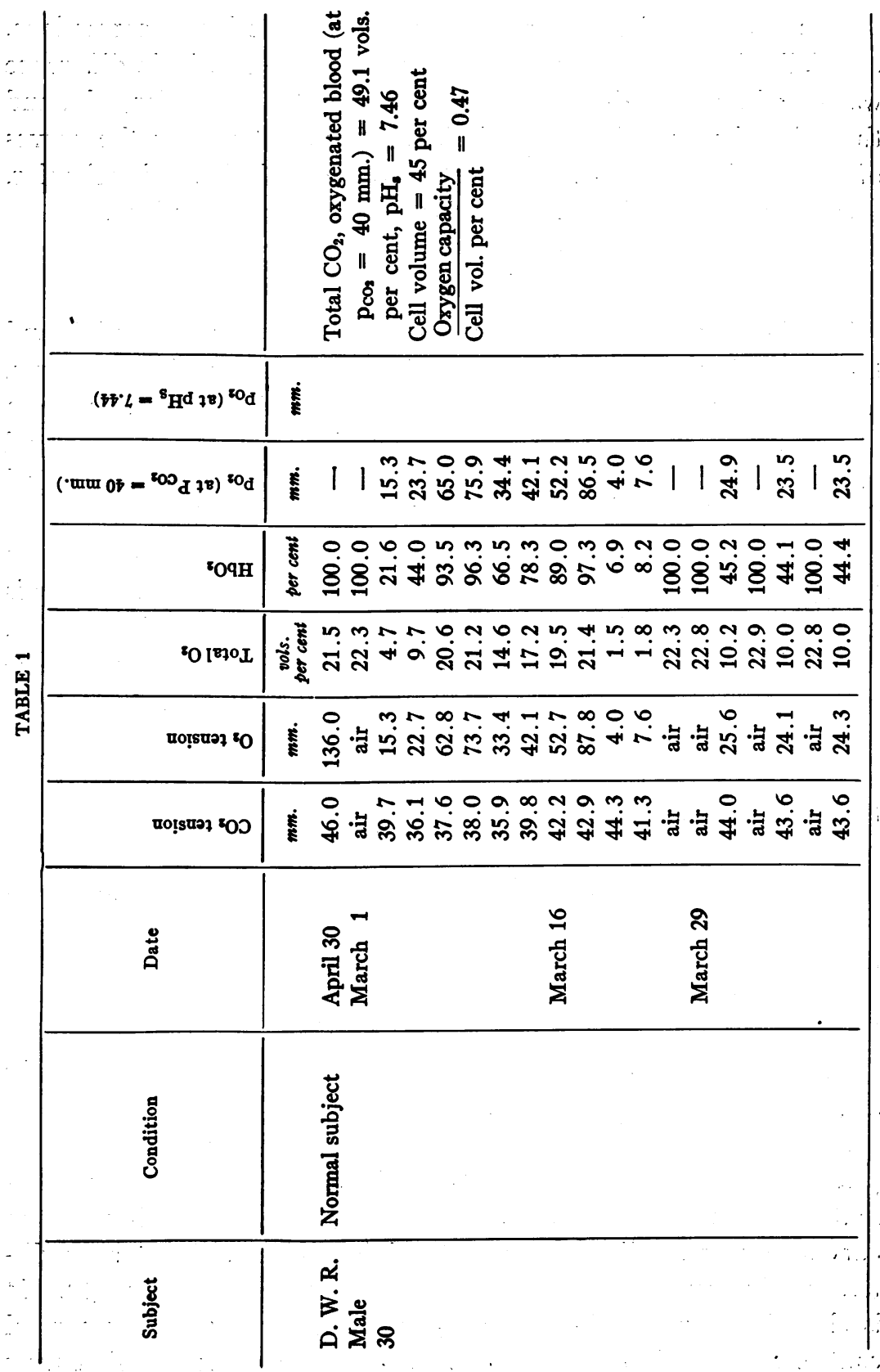


D. W. RICHARDS, JR., AND M. L. STRAUSS

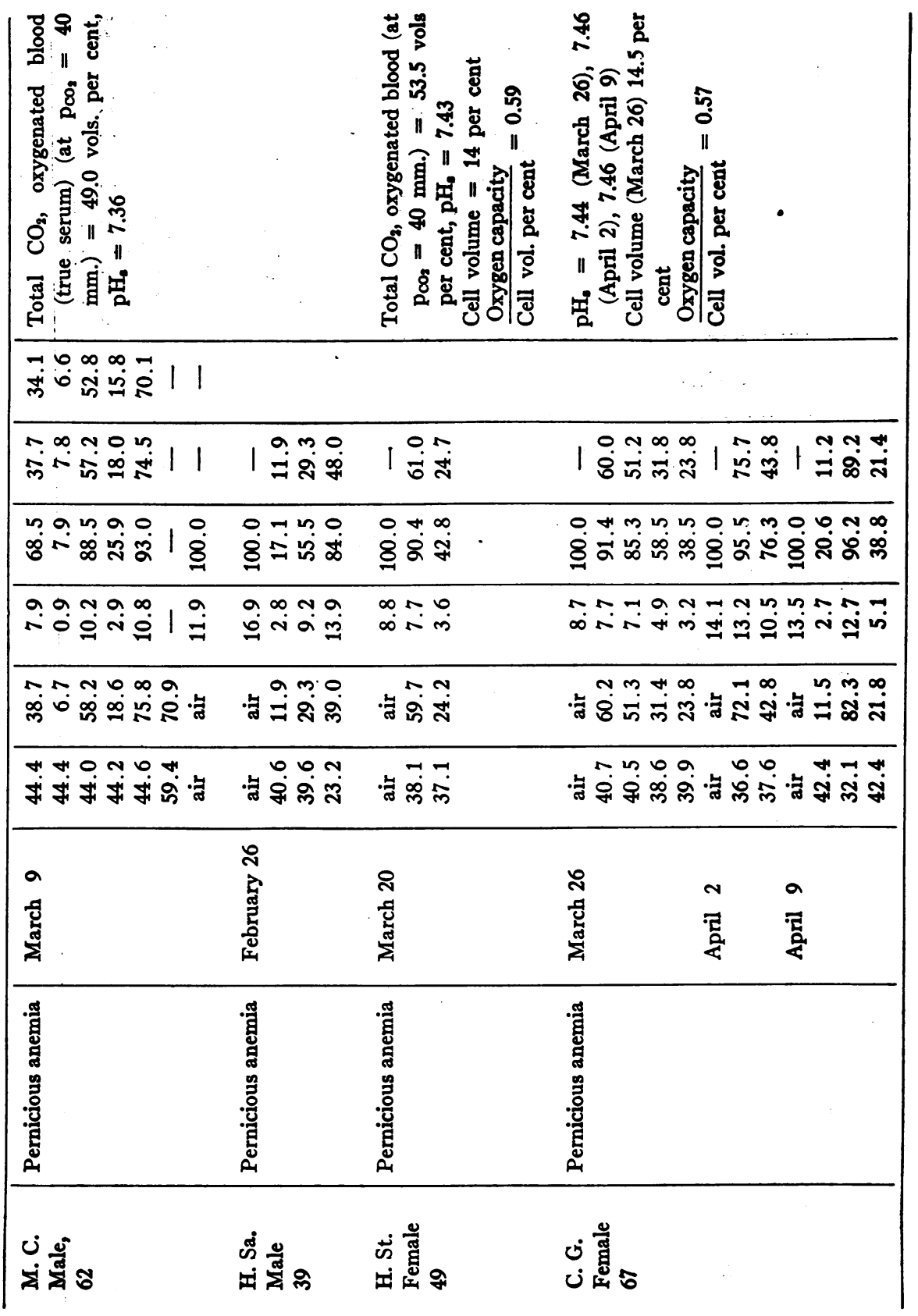




\begin{tabular}{|c|c|c|c|}
\hline • & 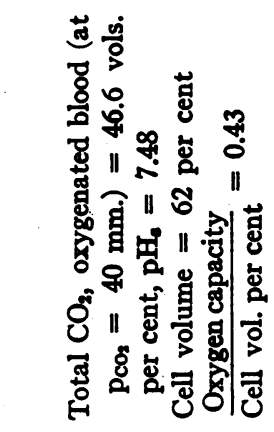 & 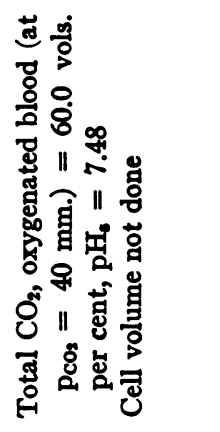 & 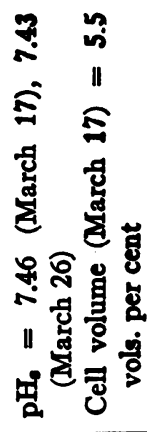 \\
\hline 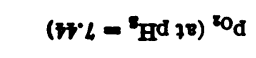 & 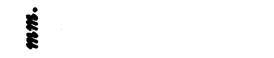 & & \\
\hline 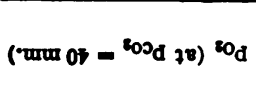 & 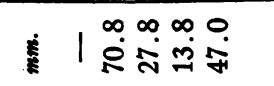 & | & 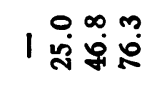 \\
\hline О्भH & 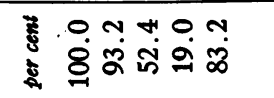 & 우웡 & 우요요 \\
\hline 8 [B] & 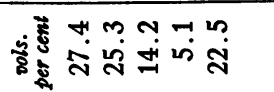 & 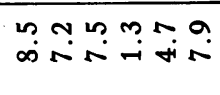 & ن \\
\hline zọ!sue? zo & 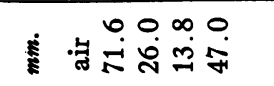 &  & 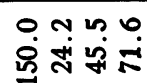 \\
\hline nọ̣suอ7 8.0J & 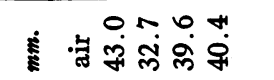 & 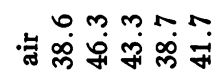 & 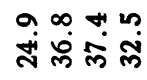 \\
\hline 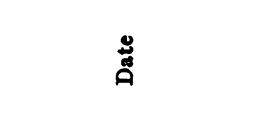 & 일 & 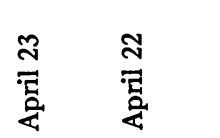 & $\begin{array}{l}\text { च } \\
\text { 总 } \\
\text { 出 }\end{array}$ \\
\hline 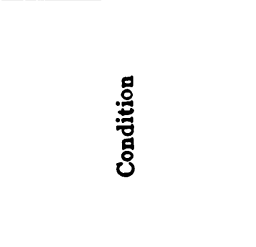 & 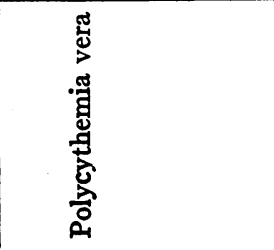 & 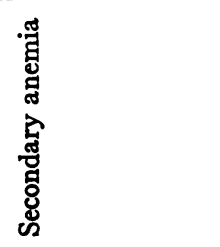 & 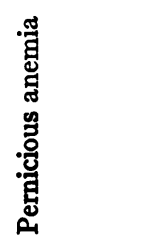 \\
\hline 苋 & 芯营 & 宊 & 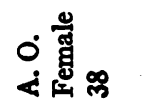 \\
\hline
\end{tabular}




\begin{tabular}{|c|c|}
\hline 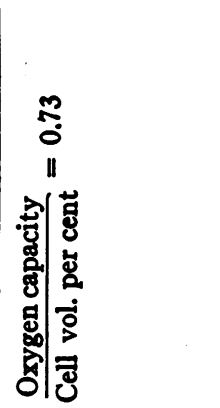 & 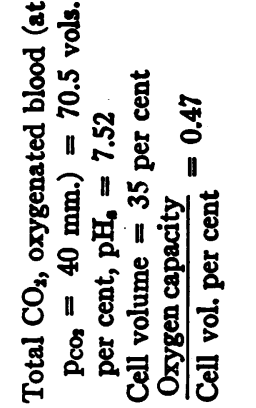 \\
\hline & | \\
\hline | & | \\
\hline 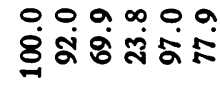 & 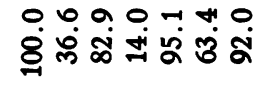 \\
\hline ن & 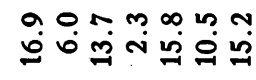 \\
\hline 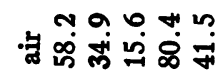 & 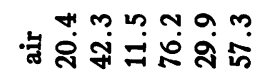 \\
\hline  & 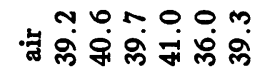 \\
\hline \multirow[t]{3}{*}{ 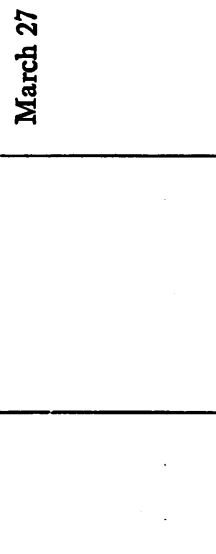 } & 疍 \\
\hline & 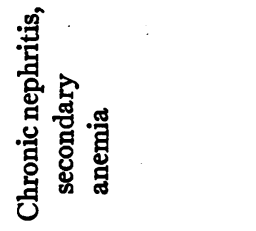 \\
\hline & ウ \\
\hline
\end{tabular}




\begin{tabular}{|c|c|c|c|}
\hline & 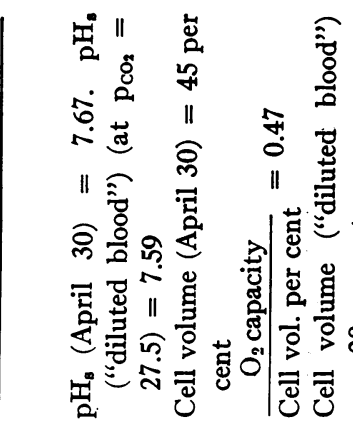 & 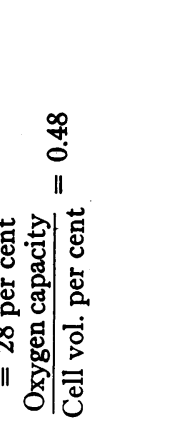 & 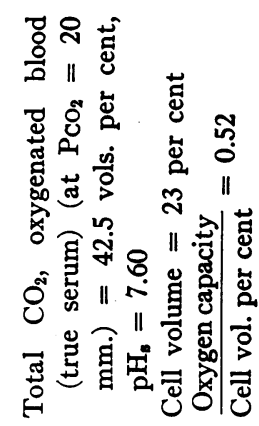 \\
\hline$\left(79^{\cdot} L={ }^{s} H^{d} \mathfrak{7 x}\right)^{z O_{d}}$ & $\dot{\Sigma}$ & $\ddot{m} \dot{8} \ddot{\circ} \ddot{a}$ & \\
\hline 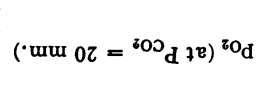 & 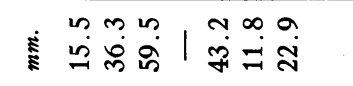 & 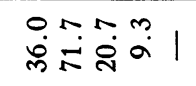 & 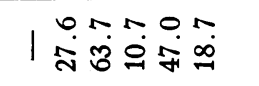 \\
\hline${ }^{8} \mathrm{O} 9 \mathrm{H}$ & 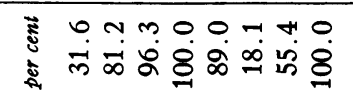 & 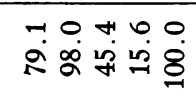 & 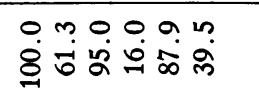 \\
\hline 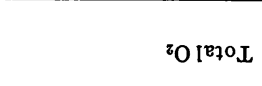 & 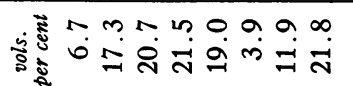 & 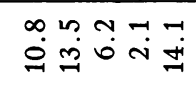 & 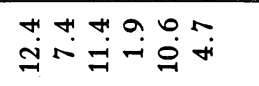 \\
\hline นоฺ̣sนว‡ ₹ 0 & 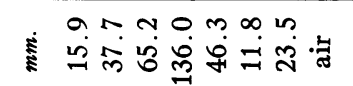 &  & 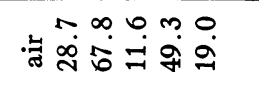 \\
\hline 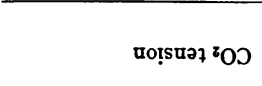 & 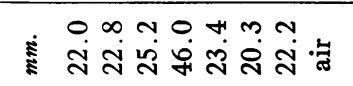 & 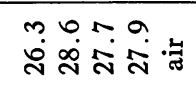 & 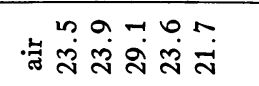 \\
\hline$\stackrel{\sharp}{\text { Ă }}$ & 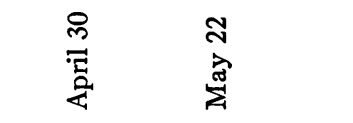 & $\stackrel{m}{\Xi}$ & 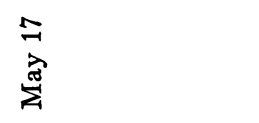 \\
\hline 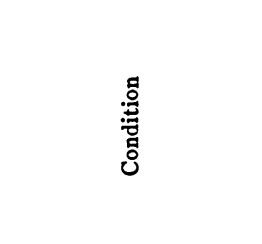 & 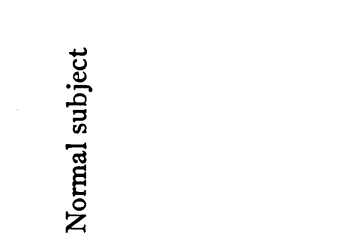 & 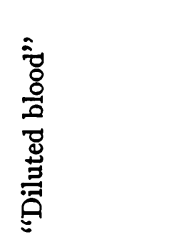 & 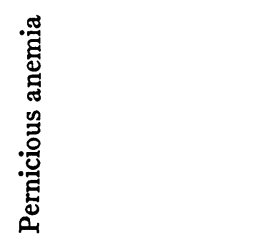 \\
\hline 䔎 & 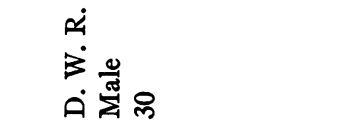 & & 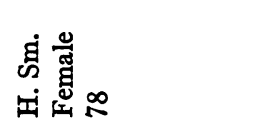 \\
\hline
\end{tabular}


D. W. RICHARDS, JR., AND M. L. STRAUSS

\begin{tabular}{|c|c|c|c|}
\hline 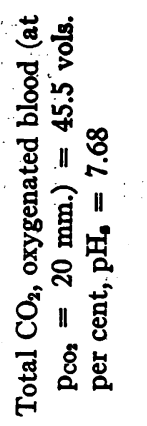 & 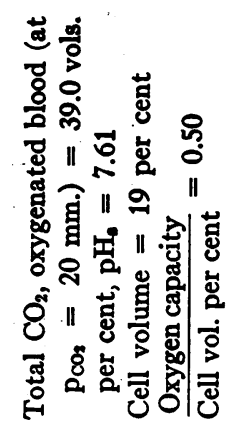 & 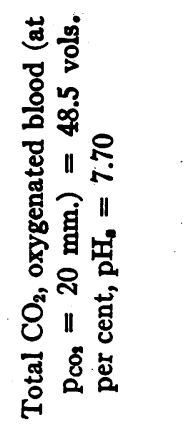 & 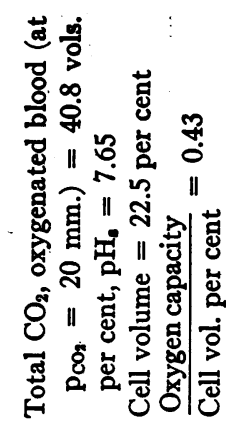 \\
\hline & \multicolumn{3}{|c|}{ 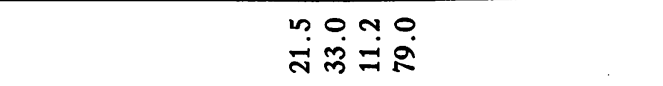 } \\
\hline | & 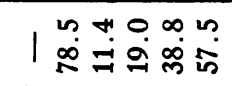 & | & 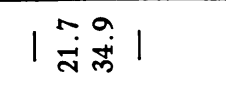 \\
\hline 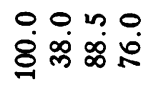 & 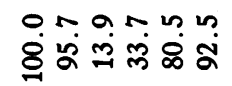 & 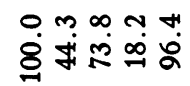 & 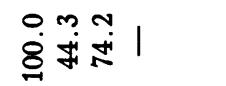 \\
\hline 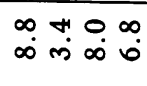 & ă & 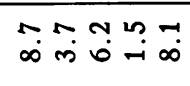 & 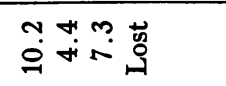 \\
\hline . & 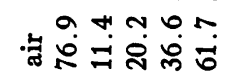 & 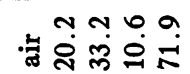 & 苞 \\
\hline 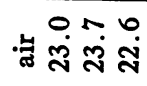 & 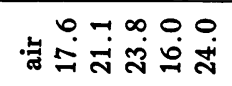 & "ส & 灵華 \\
\hline 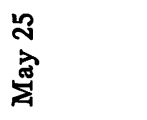 & 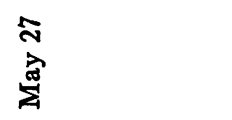 & $\begin{array}{l}a \\
\Xi \\
g\end{array}$ & 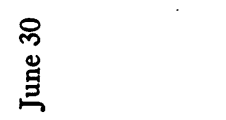 \\
\hline 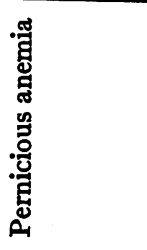 & 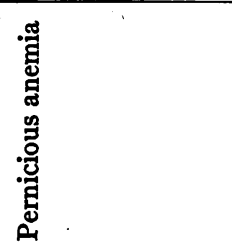 & 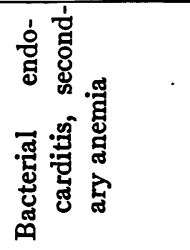 & 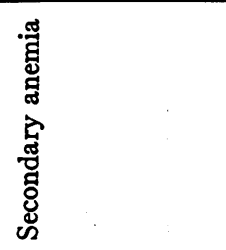 \\
\hline 丞 & 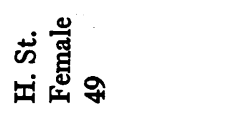 & 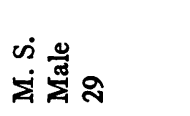 & 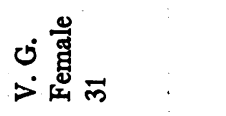 \\
\hline
\end{tabular}




\begin{tabular}{|c|c|c|c|c|}
\hline . & 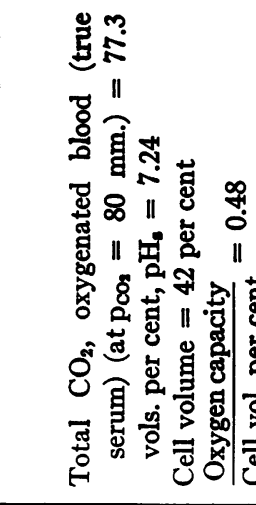 & 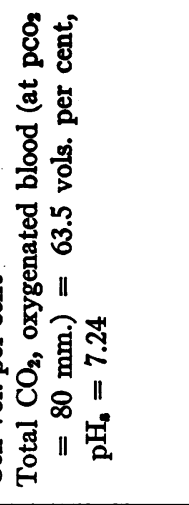 & 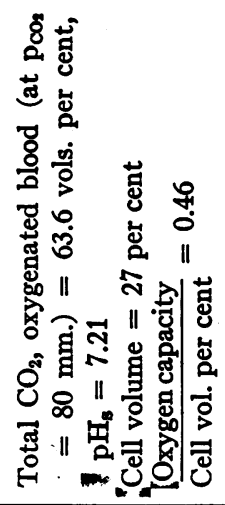 & 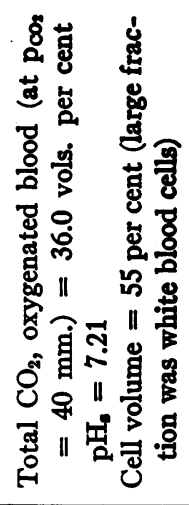 \\
\hline 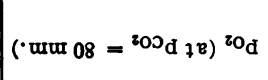 & | & 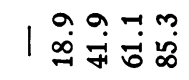 & 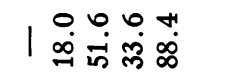 & | \\
\hline О $\mathrm{OAH}$ & 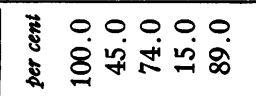 & 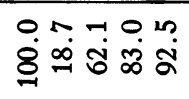 & 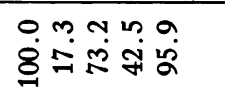 & ํำ \\
\hline${ }^{8} \mathrm{O}$ [870 L & 苟 & 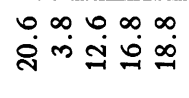 & 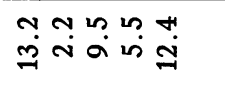 & ن̣ \\
\hline 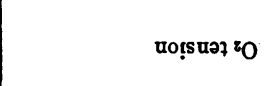 & 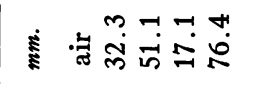 & 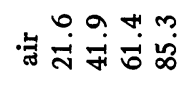 & 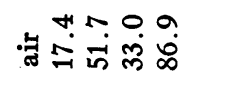 & 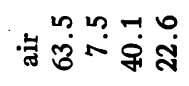 \\
\hline นо!̣sนə7 80ว & 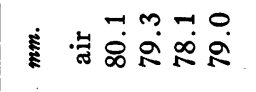 & 䒕占宅 & 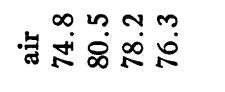 & 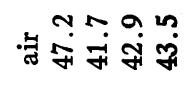 \\
\hline$\stackrel{\Xi}{\sharp}$ & $\begin{array}{l}\stackrel{?}{N} \\
\stackrel{\Phi}{\Xi} \\
\stackrel{\Xi}{2}\end{array}$ & $\stackrel{\infty}{2}$ & 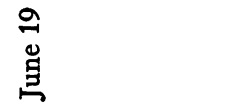 & in \\
\hline 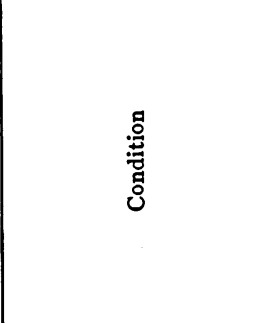 & 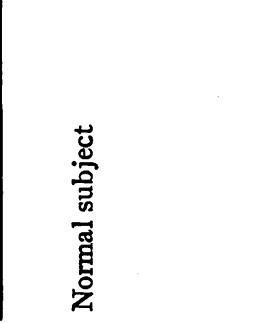 & & 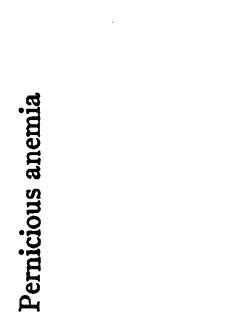 & 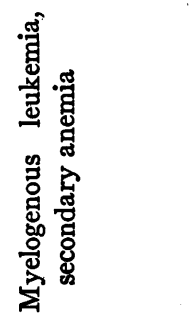 \\
\hline $\begin{array}{l}\text { 范 } \\
\text { 营 }\end{array}$ &  & & 造荧 & 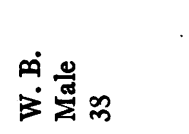 \\
\hline
\end{tabular}


are all at a definitely lower level. As far as these data are concerned, therefore, the probability is high that the condition of anemia is associated in some way with a lowering of the oxygen dissociation curve, when the $\mathrm{pH}_{\mathrm{s}}$ is in the region of 7.64 .

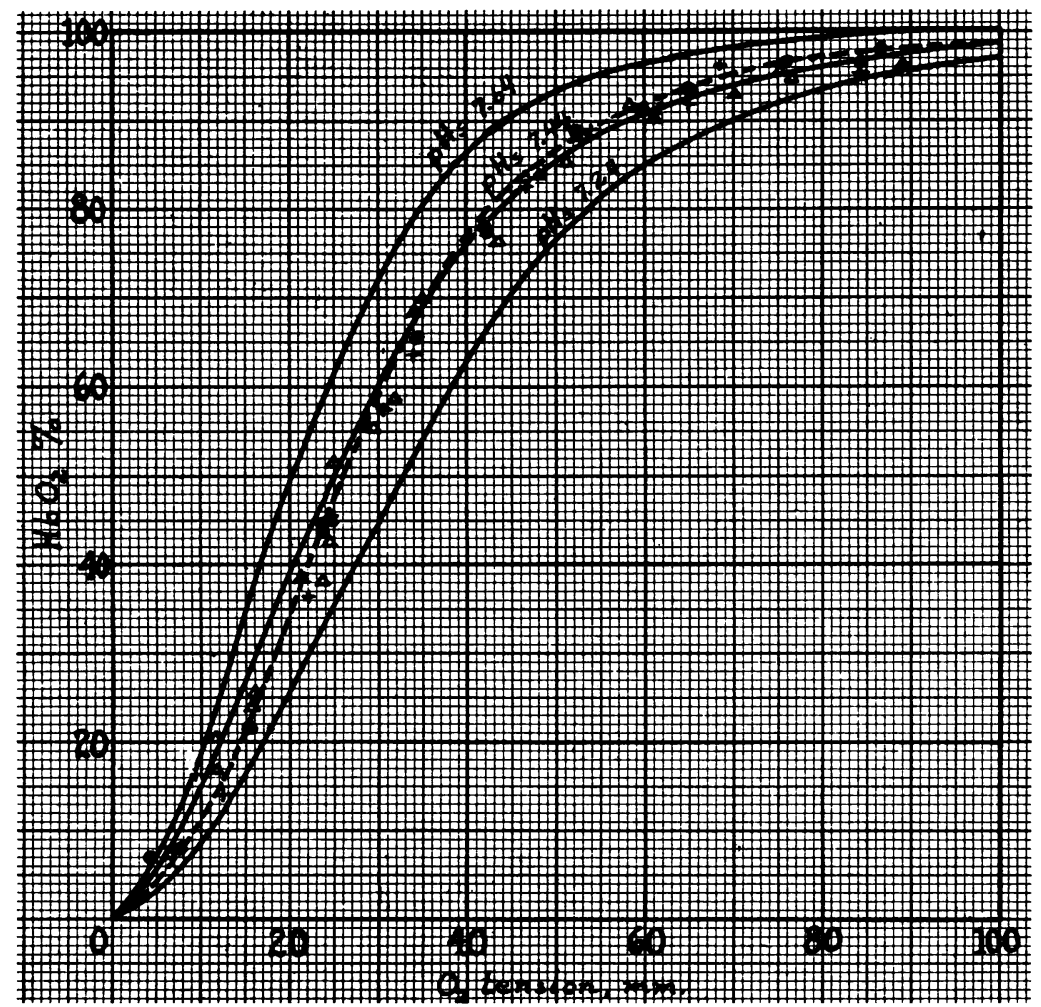

Fig. 1. Oxy-hemoglobin Dissociaticn Curves at $\mathrm{pH}_{\mathrm{s}} 7.44\left(\mathrm{CO}_{2}\right.$ Tension APPROXIMATELY 40 MM.)

Drawn curves from data of Bock, Field, and Adair. Heavy dots, normal control. Triangles $(\Delta)$, pernicious anemias. Plus marks $(+)$, secondary anemias.

This can be more clearly shown if the points are plotted logarithmically (on the principle of Hill's equation), with coördinates log $\frac{\mathrm{Hb}}{\mathrm{HbO}_{2}}$ and $\log \mathrm{p}_{\mathrm{O}_{2}}$. This has been done in figure 4 , with a curve 
OXY-HEMOGLOBIN DISSOCIATION CURVES

\begin{tabular}{|c|c|c|c|c|c|c|c|c|}
\hline 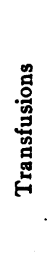 & 8 & ষ్థ \&్రి & 8 & ళ్ర & & 암용 & 8 & \\
\hline 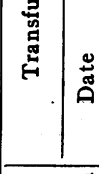 & 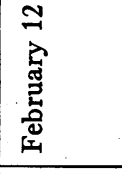 & 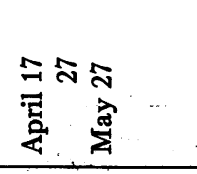 & 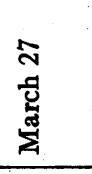 & 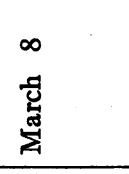 & & 足 & 突 & \\
\hline 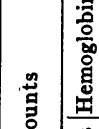 & $\stackrel{\infty}{\sim} \stackrel{2}{~}$ & 폿ำ & 88 & 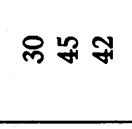 & 3 & 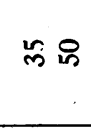 & 果 & $R$ \\
\hline 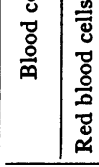 & 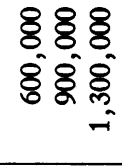 & 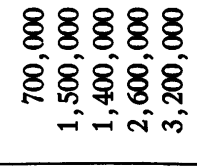 & $\begin{array}{l}88 \\
80 \\
80 \\
-1 \\
-1-1\end{array}$ & $\begin{array}{l}888 \\
88 \\
888 \\
80 \\
-10\end{array}$ & $\begin{array}{l}8 \\
8 \\
8 \\
-1 \\
-1\end{array}$ & 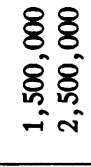 & $\begin{array}{l}8 \\
8 \\
8 \\
8 \\
8 \\
0 \\
\text { i } \\
\text { in }\end{array}$ & $\begin{array}{l}8 \\
8 \\
8 \\
\text { n. } \\
\text { n. }\end{array}$ \\
\hline 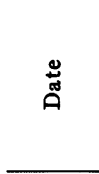 & 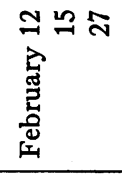 & 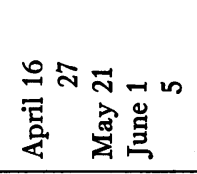 & 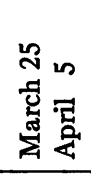 & 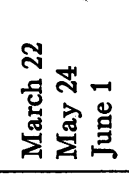 & 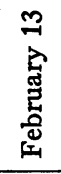 & 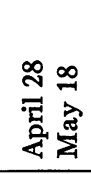 & 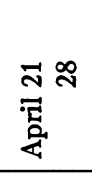 & 离 \\
\hline 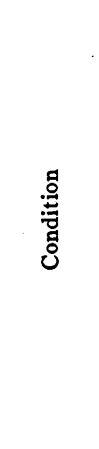 & 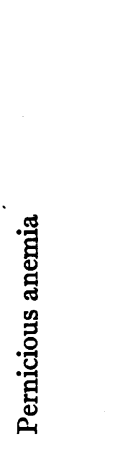 & 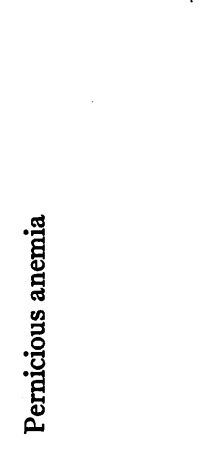 & 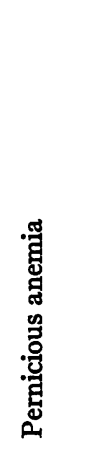 & 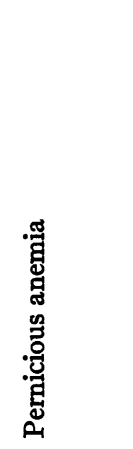 & 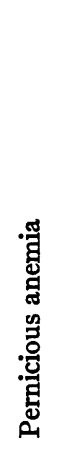 & 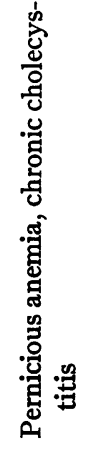 & 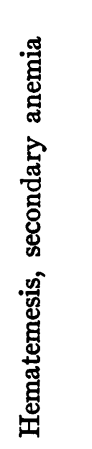 & 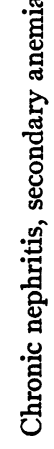 \\
\hline$\stackrel{8}{4}$ & $\widetilde{\sigma}$ & $\infty$ & $\hat{b}$ & gे & 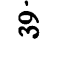 & $\stackrel{\infty}{\infty}$ & $F$ & 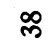 \\
\hline ڤั & $\dot{\Sigma}$ & 席 & म & मि & $\dot{\Sigma}$ & ai & ⿷匚 & $\dot{\Sigma}$ \\
\hline 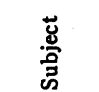 & $\begin{array}{l}\dot{\Sigma} \\
\dot{\Sigma}\end{array}$ & $\begin{array}{l}\dot{0} \\
\dot{8}\end{array}$ & ن & $\begin{array}{l}\dot{\infty} \\
\dot{\Phi}\end{array}$ & $\begin{array}{l}\text { का } \\
\text { पं }\end{array}$ & 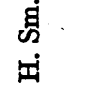 & $\stackrel{\dot{s}}{\dot{p}}$ & ن \\
\hline
\end{tabular}


D. W. RICHARDS, JR., AND. M. L. STRAUSS

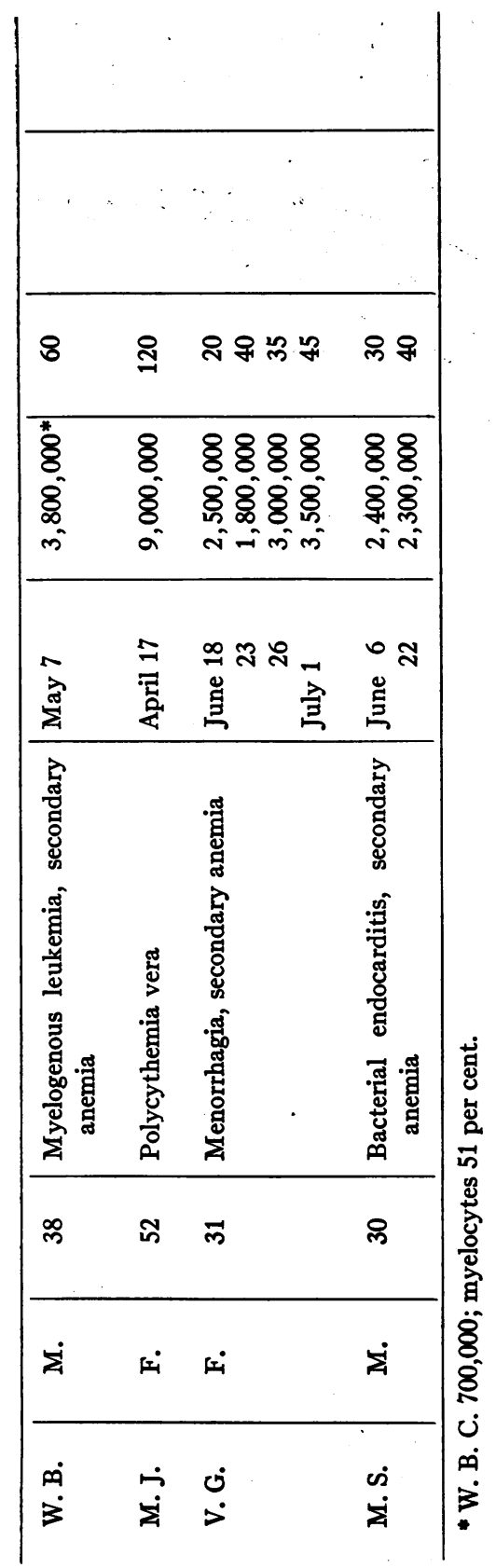


drawn through the points of normal blood, and another through those of the anemic bloods. The anemia curve is throughout its course lower than the normal.

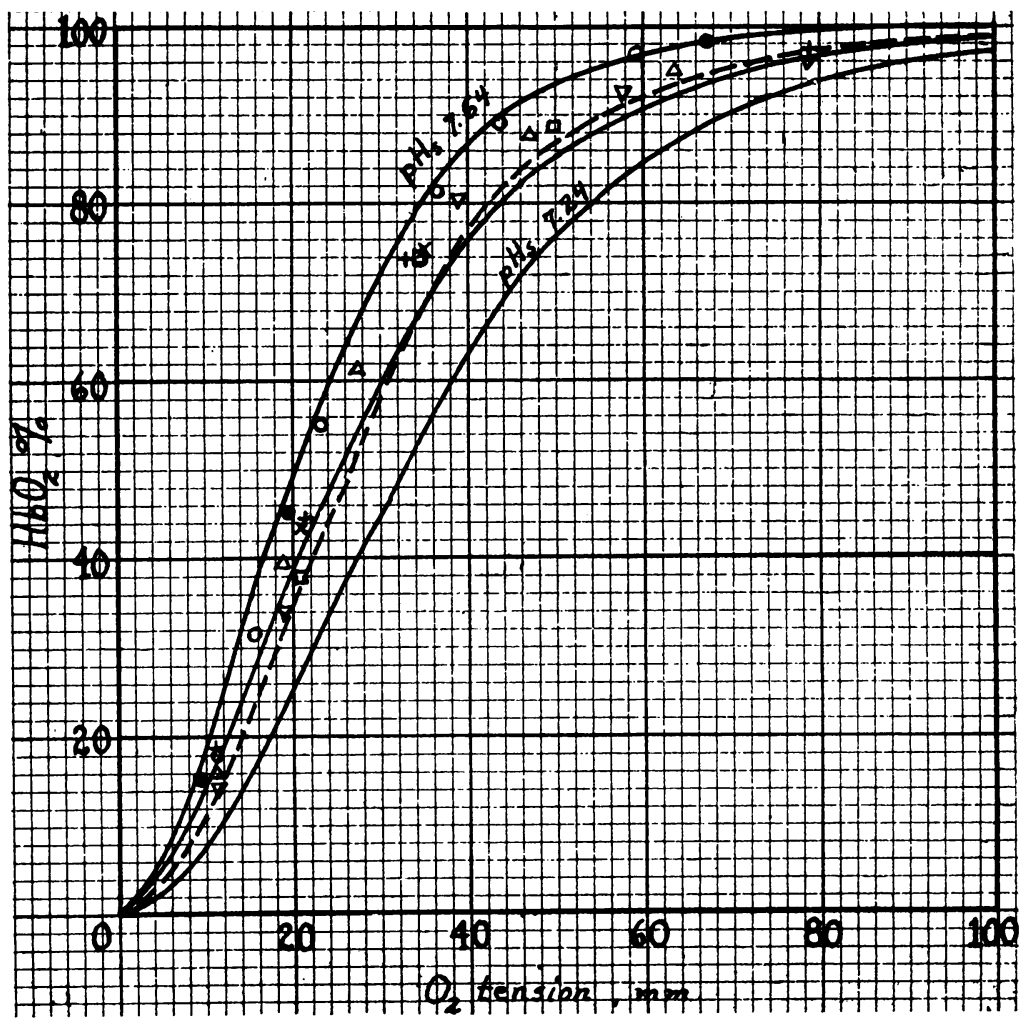

Fig. 2. Oxy-hemcglobin Dissociation Curves at $\mathrm{pH}_{\mathrm{s}} 7.64\left(\mathrm{CO}_{2}\right.$ Tension ApPRoXimately 20 mM.)

Drawn curves frcm data of Bock, Field, and Adair. Circles, normal control. Heavy dots, normal control blocd "diluted." Squares and triangles, pernicio ss anemias. Crosses and plus marks, secondary anemias.

To summarize, the data which we have obtained indicate that at $\mathrm{pH}_{\mathrm{s}}$ of 7.24 and 7.44 there are no large consistent differences between the oxy-hemoglobin dissociation curves of normal and of anemic whole bloods; but that at $\mathrm{pH}_{8}$ of 7.64 , the anemic curves are at a lower (more "acid") level than those of normal blood. 
One would not expect simple dilution of the blood with serum to be responsible for a lowering of the curves; and this was easily shown to have practically no such effect. A sample of normal

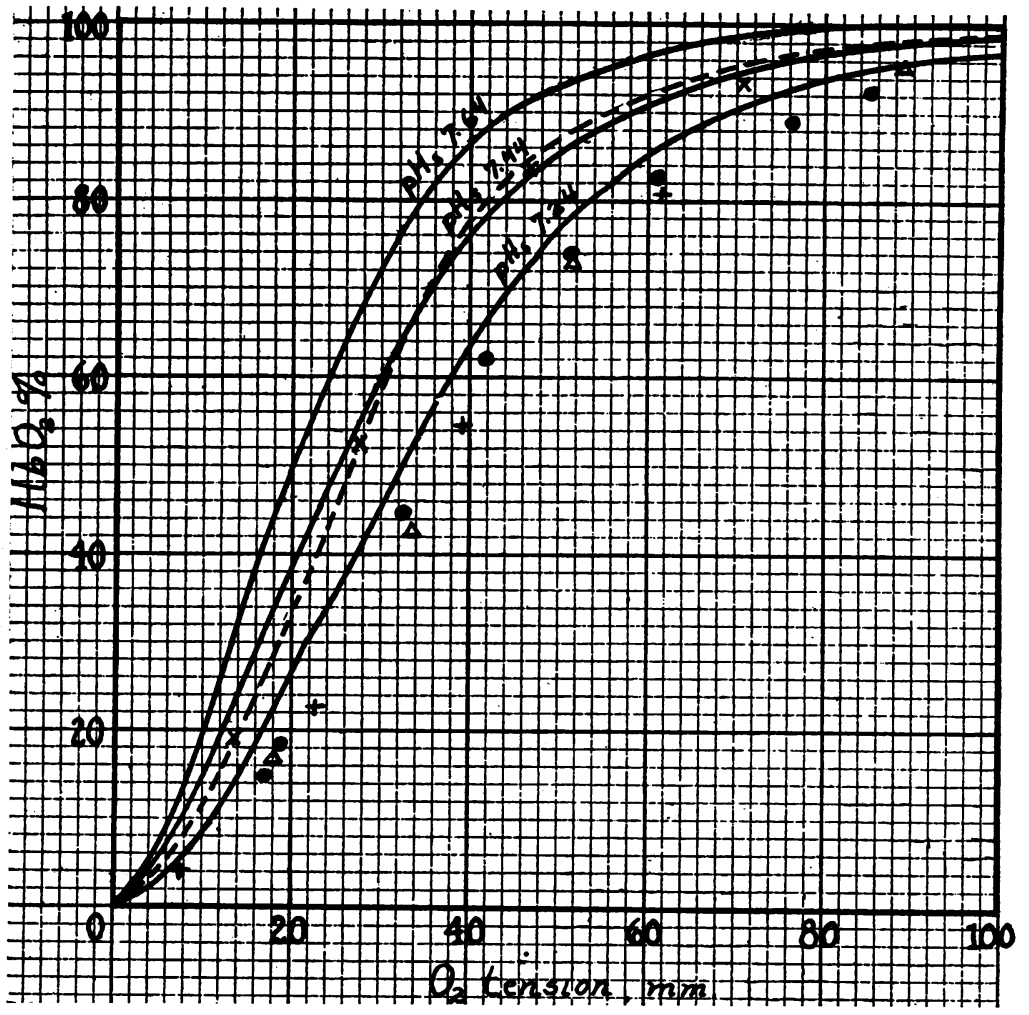

Fig. 3. Oxy-hemoglobin Dissociation Curves at $\mathrm{pH}_{8} 7.24\left(\mathrm{CO}_{2}\right.$ Tension Approximately 80 mM.)

Drawn curves from data of Bock, Field, and Adair. Heavy dots, normal control. Triangles, primary anemia. Plus marks, secondary anemia. Crosses are points on the curve of a patient with polycythemia vera, at $\mathrm{CO}_{2}$ tension of $40 \mathrm{~mm}$.

oxalated blood was centrifuged, a part of the cells mixed with the whole of the serum, and a dissociation curve determined for this diluted blood. The points, given in table 2, "diluted blood," and charted in figure 2, fell along the normal whole blood curve. 
If instead one were to assume a concentration of the hemoglobin within the blood cell, a change, of the type found in our alkaline

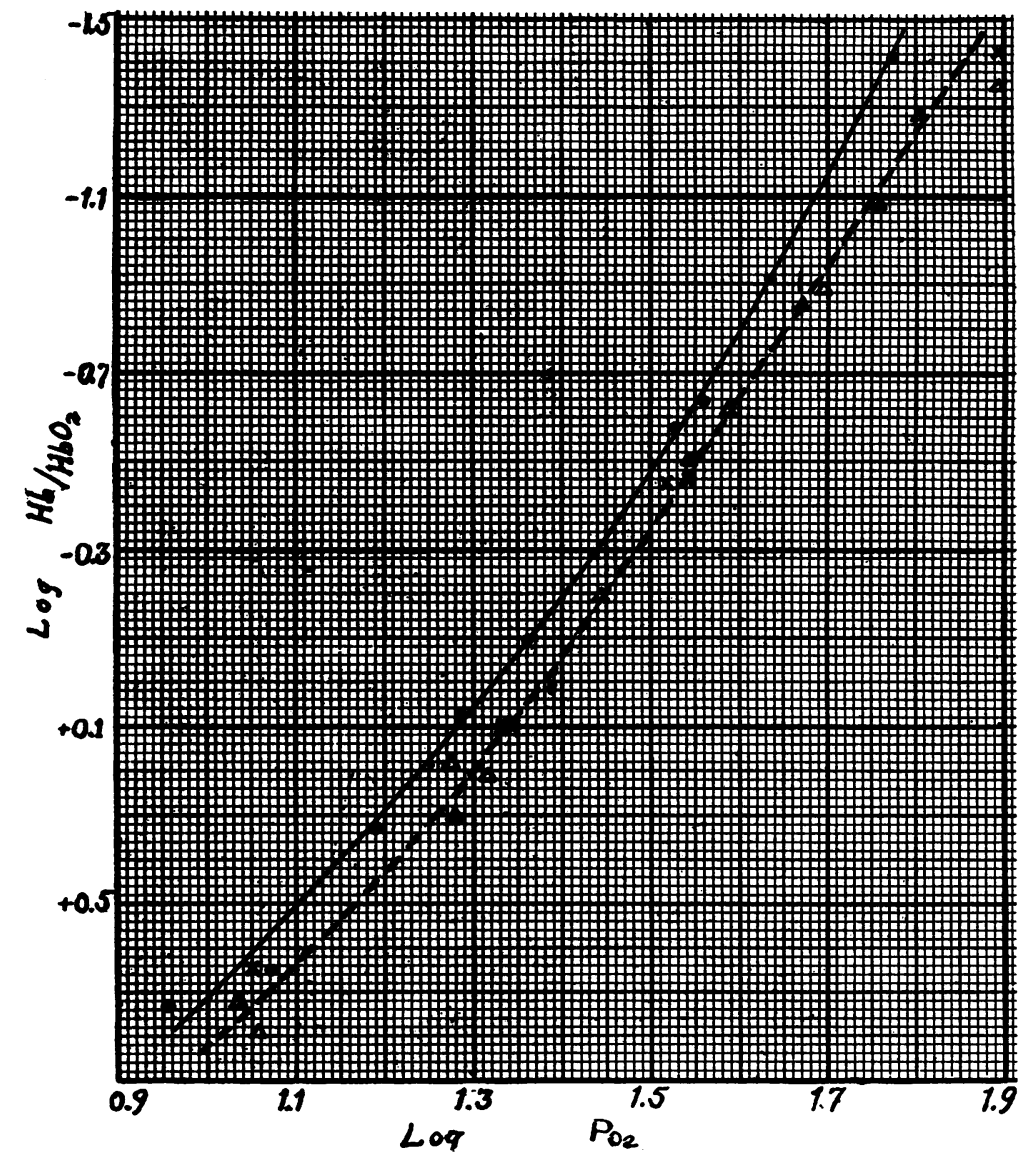

Fig. 4. Oxy-hemoglobin Dissociation Curves of Whole Blood at $\mathrm{pH}_{\mathrm{s}}$ 7.64, Plotted Logarithmically

Heavy dots and continuous curve, normal control blood. Triangles, pernicious anemias. Crosses, secondary anemias. Dotted line, average curve for anemic blood.

anemia curves, would occur, and the displacement would be greater, the more alkaline the $\mathrm{pH}_{8}$. This would be true because an increase in the hemoglobin concentration within the cell would increase the 
Donnan effect; that is, the Van Slyke $r$ would diminish and the difference between the cell $\mathrm{pH}$ and serum $\mathrm{pH}\left(\mathrm{pH}_{\mathrm{s}}-\mathrm{pH}_{\mathrm{c}}\right.$, or $\left.-\log r\right)$ would increase. Thus at equal $\mathrm{pH}_{\mathrm{s}}$ values, the $\mathrm{pH}_{\mathrm{c}}$ of the blood with increased cell hemoglobin would be lower than the $\mathrm{pH}_{0}$ of normal blood. The oxygen dissociation curve would therefore be at a lower level. Furthermore, the relative lowering would be greater the more alkaline the $\mathrm{pH}_{\mathrm{s}}$, the $\frac{-\log r}{\mathrm{pH}_{\mathrm{s}}}$ ratio having a steeper slope for blood with increased cell hemoglobin than for normal blood. This latter follows indirectly from the large increase in the dissociation of the cell protein (i.e. $[\mathrm{BP}]_{\mathrm{o}}$ ), when the solution becomes more alkaline. We have worked out these effects quantitatively using the Van

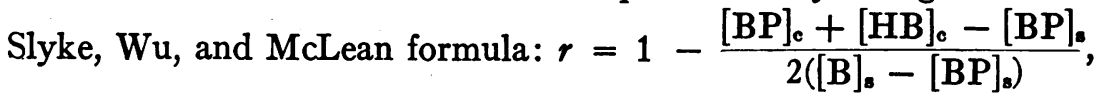
and the data from the four experiments of the same paper; assuming, for the blood with increased cell hemoglobin, values of $[\mathrm{BP}]_{\text {e }}$ and $[\mathrm{Hb}]_{\mathrm{c}} 4 / 3$ times the given normal values. These computations give consistent results in all the four experiments; an average set of values is the following from experiment 3 (oxygenated blood):

\begin{tabular}{c|c|c}
\hline \multirow{2}{*}{$\mathrm{pH}_{\mathrm{s}}$} & \multicolumn{2}{|c}{$-\log r\left(=\mathrm{pH}_{\mathrm{s}}-\mathrm{pH}_{\mathrm{c}}\right)$} \\
\cline { 2 - 3 } & $\begin{array}{c}\text { Blocd with increased cell } \\
\text { hemoglobin }\end{array}$ & Normal blood \\
\hline 7.75 & 0.32 & 0.24 \\
7.42 & 0.21 & 0.16 \\
7.08 & 0.12 & 0.10 \\
\hline
\end{tabular}

Thus, whereas at a $\mathrm{pH}_{8}$ of 7.08 , the $\mathrm{pH}_{\mathrm{o}}$ of the blood with increased cell hemoglobin is only 0.02 more acid than that of normal blood, at a $\mathrm{pH}_{\mathrm{a}}$ of 7.75 there is a difference of 0.08 . The differences are probably actually larger than those tabulated, because the $\mathrm{pH}_{\mathrm{c}}$ figures of normal blood were determined in these experiments by Van Slyke, $\mathrm{Wu}$ and McLean from the $\frac{\left[\mathrm{HCO}_{3}\right]_{\mathrm{c}}}{\left[\mathrm{HCO}_{3}\right]_{s}}$ ratios, and these ratios were considerably lower in most cases than the $r$. values as determined by the formula for $r$ given above. As we have said, we used this formula to determine the $\mathrm{pH}_{0}$ values of the blood with increased cell hemo- 
globin. That the true $\frac{\left[\mathrm{H}^{+}\right]_{\mathrm{s}}}{\left[\mathrm{H}^{+}\right]_{\mathrm{c}}}$ ratio (or, more accurately, the ratio of the activity coefficients) is not equal to $\frac{\left[\mathrm{HCO}_{3}\right]_{\mathrm{c}}}{\left[\mathrm{HCO}_{3}\right]_{\mathrm{s}}}$ but is considerably less, has been shown recently by Van Slyke, Hastings, Murray, and Sendroy (12).

The above calculations are of course only rough approximations: the formula for $r$ is itself only approximate, and the suggested changes in values of $[\mathrm{BP}]_{\mathrm{c}}$ and $[\mathrm{Hb}]_{\mathrm{c}}$ take no account of corresponding changes in other intracellular electrolyte concentrations, which would modify to some extent the percentage oxygen saturation of the hemoglobin. The figures given, however, do indicate the direction of the change that might be expected to occur, if concentration of hemoglobin within the cell were one of the conditions prevailing in a given blood. The figures also show the considerable increase in this effect when the blood becomes more alkaline.

If the concentration of hemoglobin within the cell did occur in anemia, one would expect it particularly in the primary types, with high $\frac{\text { oxygen capacity }}{\text { hematocrit }}$ ratios. Our data on this point are not very satisfactory. Hematocrit readings were obtained with the blood of two of the primary anemias at $\mathrm{pH}_{\mathbf{8}} 7.64$, giving $\frac{\text { oxygen capacity }}{\text { hematocrit }}$ ratios of 0.52 and 0.50 , as compared with 0.47 for our normal control. This difference is in the expected direction, but not large enough to account for all the lowering of these two oxygen dissociation curves. We obtained a hematocrit reading from one of the secondary anemias at $\mathrm{pH}_{\mathrm{s}} 7.64$, giving an $\frac{\text { oxygen capacity }}{\text { hematocrit }}$ value of 0.43 . This does not agree with the concentration hypothesis: the dissociation curve of this subject was lower than the normal, and yet the concentration of hemoglobin in the cell, according to the hematocrit, is less than normal. All our hematocrit values, however, are at best only approximate, as in our hands this instrument was not reliable. For this reason, it is hardly justifiable to use these values for purposes of calculation.

Determination of the electrolyte distribution in the blood of the 
subjects studied was outside the scope of this investigation: reference to the data of Peters, Bulger, Eisenman and Lee (14), showed no indication of any large, consistent variation in the electrolytes of the blood of primary or secondary anemia that might account for low oxygen dissociation curves. Gram (15) has shown that the cell chloride concentration in anemia does not vary in any regular manner with the extent of the anemia.

We wish especially to express our gratitude to Professor L. J. Henderson and Dr. C. D. Murray, in collaboration with whom this work was planned, and with whom we had the privilege of discussing our results; and to Dr. A. B. Hastings, for guidance through many technical difficulties.

\section{SUMMARY}

The oxy-hemoglobin dissociation curves of the whole blood of several subjects with anemia, primary and secondary, were investigated at serum $\mathrm{pH}$ 's of $7.24,7.44$ and 7.64 . At the lower $\mathrm{pH}$. values, all curves studied were close to curves of normal blood. At serum $\mathrm{pH} 7.64$ the anemia curves were definitely lower than the normal. One possible explanation of this fact is discussed.

Points on the curve of a patient with polycythemia vera showed no evidence of abnormality.

\section{BIBLIOGRAPHY}

1, 2. Earlier work summarized in Barcroft, J., The Respiratory Function of the Blood, Cambridge, 1914; and in Haldane, J. S., Respiration, New Haven, 1922.

3. Bock, A. V., Field, H., Jr., and Adair, G. S., Jour. Biol. Chem., 1924, lix, 353. Carbon Dioxide Dissociation Curves of Human Blood.

4. Meakins, J., Dautrebande, L., and Fetter, W. J., Heart, 1923, x, 153. The Influence of Circulatory Disturbances on the Gaseous Exchange of the Blood. IV. The Blood Gases and Circulation Rate in Cases of Mitral Stenosis.

5. Stadie, W. C., and Martin, K. A., Jour. Clin. Invest., 1925, ii, 77. The Elimination of Carbon Monoxide from the Blood.

6. Odaira, T., Tohoku Jour. Exp. Med., 1923, iv, 243. The Change in the Reserve Alkali and the Oxygen Dissociation Curve of Blood in Clinical and Experimental Anaemias. 
7. Van Slyke, D. D., Wu, H., and McLean, F. C., Jour. Biol. Chem., 1923, lvi, 765. Studies of Gas and Electrolyte Equ ilibria in the Blood. V. Factors Controlling the Electrolyte and Water Distribution in the Blood.

8. Austin, J. H., Cullen, G. E., Hastings, A. B., McLean, F. L., Peters, J. P., and Van Slyke, D. D., Studies of Gases and Electrolyte Equilibria in Blood. I. Technique for Collection and Analysis of Blood and for its Saturation with Gas Mixtures of Known Composition.

9. Adair, G. S., Jour. Biol. Chem., 1925, lxiii, 529. The Hemoglobin System. V1. The Oxygen Dissociation Curve of Hemoglobin.

10. Adolph, E. F., and Ferry, R. M., Jour. Biol. Chem., 1921, xlvii, 547. The Oxygen Dissociation of Hemoglobin and the Effect of Electrolytes upon it.

11. Adair, G. S., Barcroft, J., and Bock, A. V., Jour. Phys., 1921, lv, 332. The Identity of Hemoglobin in Human Beings.

12. Van Slyke, D. D., Hastings, A. B., Murray, C. D., and Sendroy, J., Jour. Biol. Chem., 1925, lxv, 701. Studies of Gas and Electrolyte Equilibria in Blood. VIII. The Distribution of Hydrogen, Chloride, and Bicarbonate Ions in Oxygenated and Reduced Blood.

13. Henderson, L. J., Jour. Biol. Chem., 1920, xli, 401. The Equilibrium between Oxygen and Carbonic Acid in Blood.

14. Peters, J. P., Bulger, H. A., Eisenman, A. J., and Lee, C., Jour. Biol. Chem., 1926, 1xvii, 219. Total Acid Equilibrium of Plasma in Health and Disease. V. Miscellaneous Pathologic Conditions.

15. Gram, H. C., Jour. Biol. Chem., 1924, lxi, 337. Chlorides of Serum, Blood and Corpuscles in Various Pathological Conditions. 